\title{
HECHICERÍA, BRUJERÍA Y LICANTROPÍA EN EL "Persiles" (Libro Primero)
}

\author{
Christian ANDRÈS \\ Universidad de Picardie Jules Verne (AMIENS)
}

Title: Sorcery, Witchcraft and Lycanthropy in the Persiles (Book I).

\begin{abstract}
The writer of this paper intends to examine the link that may be found between the Northern area of magic, sorcery and lycanthropy, and the Southern one, focusing on the Persiles'Book I. From this viewpoint, the feminine character of the sorceress-witch met by Rutilio in the Siena's jail and that will fly with him on a magical coat until Norway -where she will be transformed quickly into a lustful and devourer wolf- plays a fundamental and symbolic part in the connection between both worlds, the Northern one and the Southern one.
\end{abstract}

Key words: Cervantes. The Trials of Persiles and Sigismunda. Sorcery. Witchcraft. Lycanthropy.

Cada uno de los cuatro libros tiene a su hechicera. ${ }^{\mathrm{I}}$ Maurice Molho.

La magia - sea septentrional o meridional- se ve muy bien representada en el Persiles en sus cuatro libros y, a veces, con gran relieve como ocurre en el primer libro. Después de la publicación de la obra maestra que fue y es el Quijote - la primera novela moderna- el póstumo Persiles ${ }^{2}$ pudo chocar, en tiempos pasados, a varios críticos hasta granjearse cierta incomprensión de parte del gran erudito Menéndez Pelayo, por romper con la extraordinaria novedad del Quijote, por su estilo de novela bizantina, sus múltiples e increíbles peripecias, lo maravilloso y lo mágico que integran la Historia septentrional, sin hablar de la evocación de una geografía nórdica que le parecía algo fantaseada con sus numerosas islas a veces sin nombre. ${ }^{3}$

'Traducción mía; en el texto original: "Chacun des quatre Livres a sa sorcière", "Préface” de su traducción francesa y edición del Persiles, Cervantes (1994:36). No podía dejar de referirme aquí a uno de mis eminentes maestros estimados cuando era yo estudiante en la Sorbona.

${ }^{2}$ Menéndez Pelayo (1910/ 1992: 392-393). Ahí relaciona la manera de hablar de la magia en el Persiles con el envejecimiento de Cervantes ("sin duda por debilidad senil").

${ }^{3}$ Sobre islas, Andrès (1990b). 
Tampoco Julio Caro Baroja temió hablar de "una especie de regresión senil”4 en Cervantes al comparar el tono "realista, humorista y satírico" empleado antes en el Coloquio de los perros ${ }^{5}$ con el estilo, digamos idealista, usado en el Persiles.

Por suerte y con razón, se va rehabilitando desde hace cinco o seis decenios aproximadamente ${ }^{6}$ el valor experimental de semejante novela que no es solo una acertada imitación de la obra maestra de Heliodoro, sino algo más, pero que desgraciadamente sufre de su aspecto inacabado o por su acabamiento apresurado, ya que nuestro autor iba escribiendo "puesto el pie en el estribo , / con las ansias de la muerte". 7 Mas con respecto a la magia, a lo maravilloso y a la ciencia disponemos de un material abundante y lo que ahora me propongo indagar más particularmente va a ser el vínculo acaso ambiguo o resbaladizo que existe entre el campo mágico, hechiceril y licantrópico septentrional y el meridional, fijándome en el primer libro. Así que dejaré de lado lo que toca a la hechicería y a la magia en los libros siguientes: la "agarena” Cenotia en el Septentrión (II, 8), la esclava de Lorena en Provenza (III, I 5), y en Italia la judía romana (IV, 8, Io).

En el primer libro, el pensamiento mágico y las manifestaciones diabólicas parecen mejor representados, con más presencia diegética y dramatismo que en los demás libros. Puede estimarse que el principio in media res del Persiles precipita al lector en una acción insólita, en un ambiente tenebroso, nocturno, intensamente dramático con el bárbaro Corsicurvo - nombre que suena más a diablo ${ }^{8}$ que a bárbaro- extrayendo con una gruesa cuerda

${ }^{4}$ Caro Baroja (i 992: I 42).

sPiénsese en la inolvidable evocación de la bruja Cañizares.

${ }^{6}$ Para más detalles, ver la Introducción Lozano Renieblas (I 998: I I). Todo parece arrancar de la edición del Persiles por Schevill y Bonilla, en i 9 I4.

${ }^{7}$ Citaré siempre por la edición de Carlos Romero Muñoz, Cervantes (2002) Estos famosos versos van en su dedicatoria al Conde de Lemos, p. i i6. A partir de entonces incluiré el número de la página al final de cada cita en el propio cuerpo del texto.

${ }^{8}$ En efecto, en el Libro I, solo dos "bárbaros" septentrionales e isleños llevan nombre: Corsicurvo y Bradamiro. Corsicurvo está hecho con la yuxtaposición de "corsi” (corso, 
de cáñamo a una "presa” humana desde "una profunda mazmorra (p. I 27). Luego, de manera analéptica, en el capítulo siguiente, el lector descubrirá la razón diabólica y mágica por la que así se conservan "presas” humanas en la isla Bárbara apertural: una "profecía” que habría formulado el mismo demonio o "un antiguo hechicero". Esta predica la llegada de un rey conquistador del mundo y preconiza el sacrificio humano para concretarlo. La predicción supersticiosa y presentada como nórdica pedía que se hiciesen polvos los corazones de los hombres que llegasen a la ínsula y que "los diesen a beber a los bárbaros más principales de la ínsula, con expresa orden que el que los pasase, sin torcer el rostro ni dar muestras de que le sabía mal, le alzasen por su rey" (p. 138). Por lo visto, no se sabe con exactitud en qué se inspiró Cervantes, a no ser que el malicioso y sutil novelista haya mezclado varias fuentes, varios sacrificios antropofágicos. Es verdad que Carlos Romero Muñoz parece privilegiar una posible utilización por Cervantes de lecturas americanas, ${ }^{9}$ recordando la sugerencia de Schevill y Bonilla (Garcilaso de la Vega el Inca). En este caso tal sacrificio septentrional vendría a ser una suerte de proyección del mundo mágico y religioso indio sobre el mundo nórdico. Pero el mismo Carlos Romero recuerda en un Apéndice de su edición (p. 717) que se contó casi lo mismo de la reina Artemisia y las cenizas de su marido Mausolo... Además de estas sugerencias hace falta recordar lo que escribió Molho, que pudiera seguir existiendo "un recuerdo del blót" de la sangre seca ingerida por los jefes. ${ }^{\text {Io }} \mathrm{Y}$ en efecto, el blót era un sacrificio muy usado en la religión de los antiguos escandinavos y, por ejemplo, se puede leer en la Saga de Hakon el bueno, capítulo i 4 una descripción precisa de un blótveislur (banquete sacrificial) en el Throendalög noruego. ${ }^{\text {II }}$

pirata) y de "curvo", torcido. No debe olvidarse de que el diablo hace las cosas al revés, de manera torcida...

9Piénsese también en las Cartas de relación, de Hernán Cortes, López de Gomara, la Araucana de Ercilla; todo ello según Armas Wilson (i99i).

ro Véanse los Apéndices de la edición de Carlos Romero, Cervantes (2002: 71 7 ).

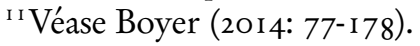


En Upsala, Adán de Brema cuenta que hacia ro7o se podía ver en un bosque colgar de las ramas cadáveres de gallos, perros, caballos, e incluso hombres. Si en el rito bárbaro que relata Taurisa no se trata de horca, sino de sacrificios humanos sin más precisión que lo del corazón hecho polvos, sin embargo considero que existe una correlación entre la palabra misma blót - con su connotación de fuerza y de sexualidad $-{ }^{12}$ y la finalidad eugenésica de semejante profecía hechiceril en la isla Bárbara: después de reconocer al bárbaro principal vencedor de la prueba ritual, será el futuro conquistador del mundo (p. 139) el hijo por nacer de su acoplamiento con la más hermosa de las jóvenes robadas o compradas. Podría ser una mera coincidencia, pero en todo caso no es de descartar tampoco un posible matiz irónico de parte de Cervantes al referirse a la mueca de asco de los competidores ingiriendo tal bebida...

Luego, por si fuera poco, aparecerá en tres ocasiones el muy antiguo tema de la licantropía. ${ }^{13}$ Se sabe que durante la Edad Media europea, se habló mucho de los hombres-lobos, ${ }^{14}$ del miedo que inspiraban, y de sus numerosas víctimas no sólo humanas. ${ }^{\text {is }}$ Habría que evocar en semejante tema el interés de los inquisidores - sobre todo el de quienes redactaron manuales de demonología- y el de médicos y juristas civiles. Recordaré la Gran Caza de brujos y brujas, la de los desgraciados acusados de tener aquel poder diabólico de transformarse en lobos y de comportarse de manera salvaje devorando hombres, mujeres y niños. Una caza que continuó en pleno renacimiento.

\footnotetext{
${ }^{12}$ Boyer (2014, I79), traducción mía.

${ }^{13}$ Libro I, capítulos quinto (pp. I69-170), octavo (pp. I88-I89) y dieciocho (pp. 243246). El tema de la licantropía ya apareció en la literatura griega con Homero y su Odisea, hace casi 2800 a. C. La maga Circe podía transformar a los hombres en lobos, pero se quedaba humano el espíritu de sus víctimas. También es de recordar la leyenda del rey Lycaon transformado en lobo por Zeus que se sintió ofendido. No se olvide que las palabras "licántropo" y "licantropía” son de origen griego, indicio de que desde la Antigüedad griega se hablaba de tal criatura real o imaginaria.

${ }^{14}$ Loups-garous en francés, varulven en escandinavo moderno.

${ }^{\text {Is }}$ Se decía que atacaban a toda clase de animales, particularmente a los rebaños de ovejas.
} 
Por otra parte si hubo poca evolución en los siglos XVI-XVII, se formuló una interpretación racionalista y científica de la licantropía, como se notará en el Persiles con el punto de vista del astrólogo Mauricio. Es decir, que coexistieron dos tendencias en la época de Cervantes, la mayoritaria adoptada por los jueces - fuesen civiles o eclesiásticos- o sea la de seguir quemando a los licántropos como seres diabólicos, y la otra, la de unos médicos que venían a coincidir con la antigua concepción de los médicos helénicos que veían en la licantropía una enfermedad real y nada más. ${ }^{16}$

El "bárbaro español” - Antonio el padre- evocará un cuadro lupino espeluznante en estos términos: "Finalmente, no sé a cabo de cuantos días y noches que anduve vagamundo por el mar, siempre más inquieto y alterado, me vine a hallar junto a una isla despoblada de gente humana, aunque llena de lobos, que por ella a manadas discurrían...” (I, s, p. I69-I70). Enseguida se destacan los elementos perturbadores siguientes: un ambiente amenazador con un mar nórdico tempestuoso, la noche y la claridad lunar, numerosos lobos feroces sobre una peña y, entre ellos, uno que habla... en castellano. ${ }^{17}$ En este pasaje, nos las habemos con una representación de lo maravilloso pagano y cristiano sin descartar una posible influencia de la Historia de gentium septentrionalium de Olao Magno. ${ }^{18}$ De hecho si se lee el capítulo 32 del libro XVIII de la Historia de las gentes septentrionales de Olao Magno, bien se ve que allí se cita la noche, "un lugar elevado" y

${ }^{16}$ Para disponer de los principales conocimientos doctrinales sobre el tema, puede consultarse con provecho el artículo muy denso (redactado en francés) de Orobitg (201 5).

${ }^{17}$ Un "licántropo bondadoso", como lo llama Lozano Renieblas (I 998: i68).

${ }^{18}$ Olaus Magnus en latín, Olaf Mansson en sueco. Fue "godo, arzobispo de Upsala, primado de Suecia y Gotia” como se precisa después del título de su famosa obra. Esta tuvo una primera edición en latín (Roma, I 55). Presenta una notable diversidad temática, con sus veintidós libros. En I 562, se publicó un Epitome, más manejable, y con I 39 grabados de gran interés. Cervantes pudo también leerla en su versión italiana : Historia delle genti... I 565. El lector español o hispanista que fuera interesado en el Septentrión -e ignorante del latín, del italiano, y del francés, ahora dispone - gracias a la labor de traductor de Daniel Terán Fierro- de la edición castellana del Epitome Historia de las gentes septentrionales (1989). 
previamente escogido, y una congregación de hombres-lobos. El lobo y el hombre-lobo están muy presentes en el mundo nórdico, ${ }^{19}$ trátese de su literatura (las sagas) o de su mitología religiosa y folclórica. En ellas, se nos habla del gran lobo Fenrir y el dios Odín -el dios mayor de la mitología nórdica - iba acompañado de dos lobos, Geri y Freki. Sólo mencionaré a los hombres-lobos de Ossory, en Irlanda, ${ }^{20}$ a la antigua islandesa Völsunga saga (S. XIII) y a los brutales y temidos guerreros nórdicos, los Berserkers. Por otra parte, debió de leer Cervantes el Jardín de flores curiosas ( I 570) de Antonio de Torquemada que ya se inspiraba en Olao Magno. El autor de la famosa miscelánea española afirma que son muy numerosos los lobos en el Septentrión, ${ }^{21}$ que existen tres suertes de lobos y se refiere a los "Neuros", o sea hombres que se transforman en lobos en ciertos periodos del año siendo la causa el pacto con el demonio, la existencia de numerosos brujos y la necromancia. Y Torquemada está convencido de la realidad de la transformación licantrópica. Su personaje Bernardo estima que ya hace mucho tiempo que "el demonio ejercita esta arte entre aquellas naciones" (p. 465), y otro personaje - Antonio- cuenta que un gallego vivía en el bosque y comía niños revestido de una piel de lobo (pp. 466-467). Luego si se cuentan pocos casos de licantropía para la península ibérica, y muchos en lo que se sabía entonces del Septentrión, concluyo que Cervantes hace en su última

${ }^{19}$ Patrizia Micozzi, después de recordar casos de licantropía en Francia, Italia, Livonia, Constantinopla, Rusia, se refiere al mundo nórdico en estos términos: "Casos de licantropía se registran también en Alemania, quizá el país más rico de hombres lobos, en Dinamarca, en Suecia, en Noruega, donde hasta corren ríos, crecen árboles y flores capaces de transmitir la enfermedad, que puede ser hereditaria o provocada por unas fórmulas mágicas, repetidas cerca del fuego y por unas hierbas, como la cicuta, el aloe, el opio, la mandrágora, el solano, el perejil, mezcladas a semillas de amapolas y a un agua especial durante el plenilunio", Micozzi (r99ı: I 29). Cfr. Donovan ( 1988, 69) quien estima que "La licantropía - la transformación en lobo- era una forma de metamorfosis atribuida a las brujas en determinadas regiones, sobre todo en Alemania y los países bálticos”.

${ }^{20}$ Giraud de Barri (Giraldo Cambrense) cuenta dicha leyenda licantrópica en su Topographia Hibernica.

${ }^{21}$ Torquemada (I982: VI, p. 462). 
novela un uso catalizador de la magia septentrional, recurriendo a ella para crear más efectismo, infundir más miedo en sus lectores, más intensidad dramática, sea con los hombres-lobos de una isla misteriosa, desierta y sin nombre en el relato del "bárbaro español" (Antonio el padre) sea con la mujer-loba en la narración del italiano Rutilio.

La segunda manifestación del tema licantrópico ocurre en el capítulo octavo del primer libro. Viene a continuación de un vuelo extraordinario sobre un manto mágico que sólo duró unas cuatro horas para ir desde la cárcel de Siena a "una tierra no conocida". ${ }^{22}$ Habría que citar todo el pasaje en cuestión y ya se sabrá que dio lugar a numerosos comentarios críticos, entre los cuales los míos. ${ }^{23}$ Es un vuelo nocturno que nos cuenta el senés Rutilio, hombre condenado por su lascivia, con una "guiadora" un poco especial, una hechicera ${ }^{24}$ sin nombre ni edad precisa por lo visto. Tal manto volador —o mágico- evocará más la magia oriental, persa o árabe que la meridional. Hace tiempo Schevill y Bonilla vieron en ello un recuerdo posible de Las Mil y una noches, con el tapiz volador del príncipe Hussain. ${ }^{25} \mathrm{Y}$ apuntan al respecto: "De cuentos orientales pasó el manto mágico al folklore de todos los países europeos. Parece que en España estaba muy divulgada la creencia según la cual cierta gente maléfica volaba por los aires con ayuda del demonio... ${ }^{26}$ De todos modos Cervantes muy bien pudo inspirarse en

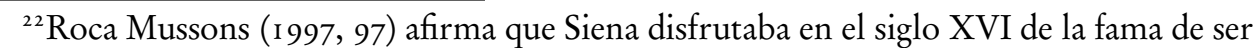
una ciudad "donde la magia tenía amplia difusión.

${ }^{23}$ Véase Andrès (1993, 532-540).

${ }^{24} \mathrm{Al}$ designar a la mujer-loba de I, 8, se emplean las palabras de "hechicera", "guiadora", "maléficas hechiceras", incluso con Mauricio, "los maléficos y encantadores", pero nunca la de "bruja". Sin embargo, el practicar el vuelo nocturno y el transformarse en loba justificarían la apelación de "bruja”.

${ }^{25}$ Véase la nota 58-i I en Cervantes (I9I4, 33 I). Allí se indica que se trata de la Historia del príncipe Abmed y de la hada Pari-Banu.

${ }^{26}$ Ibídem. También añaden a continuación ambos críticos editores del Persiles que Sancho se refiere a lo del vuelo nocturno de los brujos (Don Quijote, II, cap. XLI), y que la bruja de Berganza en el Coloquio de los perros podía hacer lo que aquí hace la bruja-loba con Rutilio. 
el Jardín de flores curiosas ya que se puede leer en él dos casos de traslado rapidísimo: uno, el de un estudiante de Guadalupe hasta Granada gracias al rocín endemoniado de un caminante en hábito de religioso; otro, contado por Bernardo y todavía más espectacular. En este se trata de "un manto grande" y volador empleado por un misterioso caminante, de un vuelo colectivo con cuatro hombres incluso con sus bestias y todos juntos viajaron muy velozmente de Olmedo a Granada... ${ }^{27}$ Así que para mí Cervantes, con el relato de Rutilio en el Persiles, reúne el mundo mágico y diabólico meridional (y más allá, oriental) con el septentrional, ya que los vuelos nocturnos tampoco escasean en las leyendas y creencias nórdicas.

Pero ahondemos algo más en semejante pasaje. En seguida llama la atención del lector el cambio rápido de postura de parte de la muy singular "guiadora”, una vez aterrizados en Noruega: comenzó a abrazar a Rutilio "no muy honestamente", notable litotes maliciosa y sensual de parte del narrador. Joaquín Casalduero vio allí la misma representación del pecado de lascivia y su castigo cristiano: "La lascivia, en la confesión de Rutilio, está envuelta en la oscuridad de la noche, en un frío desapacible y áspero". ${ }^{28}$ Bien puede ser, y en este caso será la magia de la bruja-loba en el Septentrión el mero instrumento de un castigo cristiano - y querido por Dios- por un pecado mortal cometido antes en el mediodía, en la ciudad de Siena (el pecado mortal de lujuria, por seducción y rapto de la joven hija de un caballero rico, de la que fue maestro de danzar). Ahora bien, piénsese en la fuerte relación entre Eros y Tánatos, por así decirlo. En la “construcción” de una hechicera y aún más en la de una bruja, habrá mucha frustración a la vez sexual y social. En cada evocación de las hechiceras del Persiles, damos con la fuerte atracción sexual de una mujer —ya anciana o joven no importa- ${ }^{29}$

\footnotetext{
${ }^{27}$ Torquemada (1 982, 306-308).

${ }^{28}$ Casalduero ( 1975,44$)$.

${ }^{29}$ Para limitarme al libro I del Persiles, hago constar que no se nos dice nada sobre la edad de la mujer que liberó a Rutilio de su cárcel italiana, sino que fue presa "por fatucherie”, por
} 
ejercida por un hombre, ya sea Rutilio, Antonio el Mozo o Periandro. En los tres casos narrados, el hombre deseado rechaza el amor de la hechicera y casi siempre esta conocerá un destino fatal. Me limitaré pues al caso de la mujer encontrada en la cárcel de Siena y que salvó a Rutilio de la muerte contra la promesa de casarse con ella. Se sabe que utilizó una caña mágica de la cual hablé ya en otra ocasión. ${ }^{30}$ No tiene edad -ni vieja ni joven, como si fuera la mujer (lasciva) por antonomasia. Apenas tocado el suelo noruego, parece querer cobrar el pago carnal de sus servicios anteriores. La muy sensual y cariñosa bruja no puede esperar más y abraza a Rutilio, quien trata de resistirse rechanzándola con los brazos, lo que pudiera resultar cómico si no hubiera ocurrido algo increíble, horroroso, extraordinario: “Apartela de mí con los brazos y, como mejor pude, divisé que la que me abrazaba era una figura de lobo... (p. I 88)" Nada reíble, pues, sino todo lo contrario: Cervantes quiere impresionar y espantar a su lector. "Figura de lobo", es decir que se puede comprender "figura" con el sentido de "forma", "apariencia" -y se piensa en todo un lobo- pero también con el muy común de "rostro". Lo que sugiere muy concretamente una boca y dientes amenazadores. Se sustituye muy pronto el rostro femenino de la "guiadora" - quizás agradable, no se sabe- por un rostro animal, una boca humana y sensual por otra devoradora. Es decir que nos las habemos con un símbolo teriomorfo en la terminología de Gilbert Durand. ${ }^{31}$ También hay que pensar en Jung quien interpreta el símbolo animal como la figura de la libido sexual, incluso incestuosa. ${ }^{32}$ La licantropía femenina en el Persiles se ve representada por una metonimia: una boca devoradora y mortífera de lobo o loba (¿tendrá algún sentido profundo la vacilación morfémica?). Es la metáfora del Mal

hechicería; en cuanto a Cenotia, nos enteraremos de que era una mujer de cincuenta años, es decir ya una anciana (para la época).

${ }^{30}$ Véase Andrès (1 993, 527-528).

${ }^{31}$ Durand (2016, 53-54; 7 I-72).

${ }^{32}$ Jung (I 9 1 $2 /$ I 990$)$. 
devorador y crepuscular, una breve y abortada manifestación de satanismo canibalesco. A pesar de todo Rutilio por suerte escapa a su destino fatal - verse despedazado- hincándole en el pecho a la bruja-loba un cuchillo providencial, lo que le hace perder a dicha forma a la vez su "fea" figura y la vida. Y a continuación, Rutilio cuenta lo que le dijo en toscano un hombre misterioso, que estaba en Noruega, que tuvo mucha suerte de librarse "del poder destas maléficas hechiceras, de las cuales hay mucha abundancia en estas setentrionales partes" (p. I 89), y lo de la transformación en lobos de "maléficos y encantadores", "así machos como hembras" (p. I 89). Las consideraciones siguientes muestran un fuerte contraste entre la doctrina cristiana, lo que es de creer y admitir, y la experiencia subjetiva de quien pudo o pudiera ser testigo de una transformación lupina: "Como esto pueda ser, yo lo ignoro y, como cristiano que soy católico, no lo creo; pero la esperiencia me muestra lo contrario. Lo que puedo alcanzar es que todas estas transformaciones son ilusiones del demonio y permisión de Dios y castigo de los abominables pecados deste maldito género de gente” (p. I 89). Tales consideraciones no pueden dejarnos entender lo que pensaba exactamente Cervantes de la realidad o no de la transformación licantrópica. Solo que está muy al tanto de la doctrina oficial en materia de hechicería, brujería y licantropía. Manuales de inquisidores como el famoso Malleus maleficarum ${ }^{33}$ (Martillo de las brujas) le eran evidentemente conocidos. La última frase insiste en la ilusión diabólica - que es la tesis doctrinal- si viene después de la hipótesis de una realidad física de la transformación lupina. Más adelante en el capítulo dieciocho del primer libro, Cervantes volverá sobre el tema licantrópico, prudentemente, es decir a la vez de manera doctrinal y científica mediante el diálogo entre el astrólogo Mauricio y el príncipe danés Arnaldo. Frente a la credulidad de Arnaldo y Rutilio, el racionalista Mauricio da

${ }^{33}$ Por dos dominicos Sprenger (Iacobus) e Institor (Henricus), en I 486- I 487 (Estrasburgo). Se publicaron unas treinta ediciones entre i 487 y 1669. 
una explicación médica y científica a la licantropía o locura lupina. Se nos dice que es una verdadera enfermedad, una locura que provoca en el hombre afectado la ilusión de convertirse en lobo y un comportamiento semejante al de una fiera. Hablando así Mauricio, nos recuerda la opinión médica de Johann Wier ( I 5 I 5-I 588 ) quien explica en su De praestigiis daemonum (I 563 ) que los hombres-lobos no tienen nada que ver con demonios, sino con enfermos mentales, melancólicos y que en vez de castigarlos o matarlos, mejor sería curarlos. Y como prueba de lo que afirma, Mauricio se refiere a la existencia de los lobos “menar” en Sicilia, la mayor isla mediterránea. Insiste en el papel de la imaginación en la licantropía si Rutilio vuelve a su escepticismo existencial: "No sé (...); lo que sé es que maté la loba y hallé muerta a mis pies la hechicera" (p. 246). Y Mauricio concluye categóricamente en tal materia brujeril y licantrópica: “(...) y quede desde aquí asentado que no hay gente alguna que mude en otra su primer naturaleza" (p. 246). Como ya se negó la metamorfosis con todo vigor pensando en San Agustín (Ciudad de Dios, XVIII, I 8), el Canon episcopi (hacia 900) y Santo Tomás de Aquino.

Cervantes supo servirse magistralmente de la materia mágica en su Persiles como lo hizo antes en otras obras suyas, principalmente en el Coloquio de los perros, aunque lo hiciera de otra manera y con mejor apreciación de la crítica en general. Aquí pudo relacionar muy hábilmente la magia septentrional con la magia meridional sobre todo mediante la mujer hechicera y bruja - sin nombre ni edad precisa- que aparece en una cárcel de Siena para regresar muy velozmente a Noruega en un manto volador y transformarse (según Rutilio) en un lobo o loba. En el Persiles pues, se manifiestan prácticas de magia negra, ya vinieran del Sur o del Norte de Europa. Y Cervantes refuerza aparentemente la fascinación por lo maravilloso y lo diabólico principiando su Persiles en un mundo septentrional nocturno, frío y violento desde las primeras líneas con la evocación tétrica de la isla Bárbara y sus sacrificios rituales que muy bien pudieran aludir al blót de los 
antiguos escandinavos. La hechicera-bruja encontrada en una cárcel italiana capaz de metamorfosearse en loba lúbrica y antropofágica, sea de origen italiano o noruego - no se sabe- será una manifestación diabólica que establece un vínculo entre la parte septentrional y la parte meridional de la novela cervantina. ${ }^{34}$ Esta por su parte se relaciona con el inconsciente colectivo, ciertos arquetipos, la sexualidad, la muerte y ciertas frustraciones individuales y sociales. Parece decirnos Cervantes que el Mal, lo diabólico es algo universal. En el campo mágico compruebo que Cervantes en varias obras suyas y hasta la última - el Persiles - se muestra curiosamente atraído por el tema hechiceril y brujeril, una cuestión por fin bastante compleja. ${ }^{35}$ Pero al mismo tiempo el gran novelista queda algo ambiguo en la confrontación de las opiniones de sus personajes. Sin duda para que cada lector se sienta libre de formarse su propia opinión en asunto tan peligroso.

\section{BIBLIOGRAFÍA}

Andrès, Christian (I990a), "Figures de la Sorcière chez Cervantès et Lope de Vega", Iris, III, 8-9, pp. 89-109.

Andrès, Christian (1990b) Insularidad y barbarie en Los trabajos de Persiles y Sigismunda", Anales Cervantinos, XXVIII, pp. Iо9-I 23.

AndRÈs, Christian (1993), "Fantasías brujeriles, metamorfosis animales y licantropía en la obra de Cervantes", José María Casasayas (coord.), Actas del III Coloquio Internacional de Cervantistas (Alcalá de Henares, 1990), Barcelona: Anthropos, pp. 527-540.

AndRÈs, Christian (1997), "Erotismo brujeril y hechicería urbana en Los trabajos de Persiles y Sigismunda", Anales Cervantinos, XXXIII, pp. 165-175.

Andrès, Christian (2006), "Brujería”, Gran Enciclopedia Cervantina, Madrid: Editorial Castalia, II, pp. Is I3a-is2 ia.

Armas Wilson, Diana de (1991), Allegories of Love: Cervantes" "Persiles and Sigismunda", Princeton: Princeton University Press, I99I.

Boyer, Régis (2014), Le monde du double / La magie chez les anciens Scandinaves, Paris: Berg International.

Caro Baroja, Julio (1992), Vidas mágicas e Inquisición, Madrid: Ediciones Istmo, vol. I.

Casalduero, Joaquin (1975), Sentido y forma de Los trabajos de Persiles y Sigismunda, Madrid: Editorial Gredos.

Cervantes Sanvedra, Miguel de (i9i4), Obras completas. Persiles y Sigismunda, R. Schevill y A. Bonilla, eds., Madrid: Imprenta de Bernardo Rodríguez.

Cervantes Sanvedra, Miguel de (1994), Les Travaux de Persilles et Sigismonde, (1994), Maurice Molho ed. y trad, Paris: Librairie José Corti.

${ }^{34}$ También a su modo la "agarena" Cenotia, pero sin la dimensión excepcional que cobra la licantropía.

${ }^{35}$ Véase Molho (1992) Y Lara Alberola (2008, I76-178). 
Cervantes Sanvedra, Miguel de (2002), Los trabajos de Persiles y Sigismunda, Carlos Romero Muñoz, ed., Madrid: Cátedra.

Cervantes Sanvedra, Miguel de (20 1 8), Los trabajos de Persiles y Sigismunda, Madrid: Real Academia Española.

Donovan, Franck (1988), Historia de la brujería, Madrid: Alianza Editorial.

Durand, Gilbert (2016), Les structures anthropologiques de l'imaginaire, (ra. ed.: I 969, Paris: Bordas), Paris: Dunod.

Jung, Karl Gustav (1990), Metamorfosis y símbolos de la libido, publicado en Símbolos de transformación, Barcelona: Paidos ( ${ }^{\mathrm{a}}$ ed.: en alemán: 19 I 2, Symbole der Wandlung, Zurich: Rascher, ed.).

Lara Alberola, Eva (2008), "Hechiceras y brujas: algunos encantos cervantinos", Anales Cervantinos, XL, pp. I45-179.

Lozano Renieblas, Isabel (1998), Cervantes y el mundo del Persiles, Alcalá de Henares: Centro de Estudios Cervantinos.

Magno, Olao (1 989), Historia de las gentes septentrionales, Daniel Terán Fierro, trad. y ed., Madrid: Editorial Tecnos ( 1 a. ed. en latín: Olaus Magnus, Historia de gentium septentrionalium, Roma, I 555 ).

Menéndez Pelayo, Marcelino (1992), Historia de los heterodoxos españoles, II, I $8^{\mathrm{a}}$ ed., Madrid: CSIC ( a $^{\text {e }}$ ed.: i 880, Madrid: Librería católica de San José).

Micozzi, Patrizia (I991), "Tradición literaria y creencia popular: el tema del licántropo en Los trabajos de Persiles y Sigismunda, de Cervantes”, Quaderni di Filologia e Lingue Romanze, 6, pp. IO7-I 52.

Моцно, Maurice (1992), "El sagaz perturbador del género humano”, Bulletin of the Cervantes Society of America, I 2, pp. $2 \mathrm{I}-32$.

Orobit G, Christine (2015), "La Lycanthropie dans la prose doctrinale du XVIe et du XVIIe siècle", Bulletin Hispanique, t. I 17, 2, pp. 549-568.

Roca Mussons, Maria A. (1997), Contrapuntos cervantinos, Firenze: Alinea Editrice.

Sprenger, Jacques e Institoris, Henry (I 973), Le Marteau des Sorcières, Paris: Plon (Malleus Maleficarum, Ia. ed.: I486-1 487, Strasbourg: Jean Prüss aîné.

Torquemada, Antonio de (1 982), Jardín deflores curiosas, ed. de Giovanni Allegra, Madrid: Castalia (ra. ed.: Salamanca, I 570).

recibido: junio de 2018 aceptado: octubre de 2018 
